\title{
Efectividad en el aprendizaje de la punción venosa en estudiantes de enfermería utilizando dos estrategias didácticas: “Una experiencia investigativa en aula"1
}

Fanny Esperanza Acevedo Gamboa²

Juan Carlos Díaz Álvarez ${ }^{3}$

Juan Carlos Cobo Gómez

doi:10.11144/Javeriana.ie18-2.eapv

Cómo citar: Acevedo Gamboa FE, Díaz Álvarez JC, Cobo Gómez JC. Efectividad en el aprendizaje de la punción venosa en estudiantes de enfermería utilizando dos estrategias didácticas: "Una experiencia investigativa en aula”. Investig Enferm. Imagen Desarr. 2016;18(2):61-75. http://dx.doi.org/10.11144/Javeriana.ie18-2.eapv

1. Artículo original de investigación. Fecha de recepción: 25 de marzo de 2015. Fecha de aceptación: 7 de diciembre de 2015.

2. Profesora asistente del Departamento de Enfermería Clinica, Facultad de Enfermeria, Pontificia Universidad Javeriana, Bogotá, Colombia. Correo electrónico: facevedo@javeriana.edu.co

3. Profesor asistente del Departamento de Enfermería Clínica, Facultad de Enfermería, Pontificia Universidad Javeriana, Bogotá, Colombia. Correo electrónico: j.diaza@javeriana.edu.co

4. Asistente Vicerrectoría de Investigación y profesor hora cátedra de la Facultad de Ciencias Económicas y Administrativas, Pontificia Universidad Javeriana, Bogotá, Colombia. Correo electrónico: cobo-j@javeriana.edu.co 


\section{Resumen}

Introducción: La punción venosa es un procedimiento asistencial que requiere entrenamiento por repetición sustentado en estrategias didácticas para generar habilidad operativa e instrumental y habilidad de pensamiento de orden superior incluida la comparación, el contraste y la transferencia del conocimiento. Objetivo: Comparar la efectividad de dos estrategias didácticas a través de la simulación clínica: "modelado docente" y "guía de procedimiento para el aprendizaje autónomo de la punción venosa" en un grupo de estudiantes de tercer semestre de enfermería en una institución de educación superior. Método: Estudio cuantitativo, observacional y evaluativo de investigación en aula, con 175 participantes, que comparó muestras con variables aleatorias, obtenidas en cinco periodos académicos entre el 2012 y el 2014, el cual buscó evaluar la diferencia establecida en las varianzas de las pruebas aplicadas. La información fue recolectada a través de la utilización de una lista de chequeo validada y el análisis de los datos fue realizado con la prueba F de Fisher. Resultados: En algunos periodos académicos, la estrategia de modelado docente presentó mejor comportamiento en el aprendizaje; en otros periodos académicos, la estrategia de la guía de procedimiento para el aprendizaje autónomo mostró mayor desempeño y al contrastar los resultados obtenidos en algunos periodos académicos no hubo diferencia significativa en las estrategias didácticas utilizadas. Conclusiones: Se demostró que ambas estrategias didácticas favorecen el aprendizaje de la punción venosa; sin embargo, la estrategia "guía de procedimiento para el aprendizaje autónomo" ofrece la misma efectividad si se utiliza sola o combinada con la estrategia "modelado docente".

Palabras clave: estrategias; aprendizaje; guía; simulación; estudiantes de enfermería

\section{Effectiveness in the Learning of Venipuncture in Nursing Students, using Two Didactic Strategies: "An Investigative Experience in Class"}

\section{Abstract}

Introduction: Venipuncture is a care procedure that requires training by repetition, supported by didactic strategies to generate operative and instrumental ability as well as superior order thinking abilities, including comparison, contrast and knowledge transference. Objective: To compare the effectiveness of two didactic strategies through clinical simulation: "teacher modeling" and "procedure guide for autonomous learning of venipuncture" in third semester nursing students in a university. Methodology: Quantitative, observational and evaluative study of class investigation, with 175 participants, that compared samples with random variables, obtained in five academic periods between 2012 and 2014, and that aimed to evaluate the differences established in the variances of applied tests. The information was recollected through a check list previously validated, and the data analysis was made with F-Fisher test. Results: In some academic periods, the strategy of teacher modeling presented better behavior in learning. In other academic periods, the strategy of procedure guide for the autonomous learning showed better performance; and when results in some academic periods were contrasted, there was no significant difference in the didactic strategies used. Conclusions: It was demonstrated that both didactic strategies helped the learning of venipuncture, although the strategy "procedure guide for autonomous learning" offered the same effectiveness if it was used alone or combined with the strategy "teacher modeling".

Keywords: strategies; learning; guide; simulation; nursing students 


\section{Efetividade no aprendizado da punção venosa dos estudantes de enfermagem com a utilização de estratégias didáticas: "uma experiência investigativa na sala de aula"}

\section{Resumo}

Introdução: A punção venosa é um procedimento assistencial que requer treinamento repetitivo amparado em estratégias didáticas de ensino, que gerem habilidades e capacidades operacionais e instrumentais e habilidades de pensamento de ordem superior que abranjam a comparação, o contraste e a transferência de conhecimento. Objetivo: Comparar a efetividade de duas estratégias didáticas de ensino por meio de simulação clínica: "modelagem feita pelo professor" e "aprendizagem independente com guia de procedimentos para punção venosa", em estudantes de terceiro semestre de enfermagem numa instituição de ensino superior. Materiais e métodos: Foi realizado um estudo de pesquisa observacional quantitativo e avaliativo realizado em sala de aula, com 175 participantes, para comparar amostras com variáveis aleatórias obtidas em cinco períodos acadêmicos entre os anos 2012 a 2014, o estudo avalia diferenças estabelecidas nas variâncias das provas aplicadas. As informações foram coletadas através do uso de uma lista de verificação previamente validada. A análise dos dados foi realizada com o teste de F-Fisher. Resultados: Em alguns periodos acadêmicos, a estratégia de modelagem feita pelo professor apresenta melhor comportamento no aprendizado, em outros períodos acadêmicos, a estratégia de aprendizagem independente com guia de procedimentos para punção venosa apresentou um melhor desempenho. Ao fazer a relação dos resultados obtidos entre alguns períodos acadêmicos, não se apresentaram diferenças estatísticas nas estratégias didáticas utilizadas. Conclusões: Foi demonstrado que as duas estratégias de ensino promoverão o aprendizado de punção venosa, no entanto, a estratégia "guia de procedimentos para a aprendizagem independente" oferece a mesma efetividade quando usada sozinha ou combinada com a estratégia "modelagem feita pelo professor".

Palavras chave: estratégias; de aprendizagem; de orientação; de simulação; os estudantes de enfermagem 


\section{Introducción}

La implementación de estrategias centradas en el aprendizaje requiere ingenio y creatividad, a efectos de fomentar autoformación y autonomía en el estudiante (1); por ello, la simulación clínica se ha convertido en una de las estrategias de aprendizaje necesarias en la formación de los profesionales de la salud, pues precisa didácticas innovadoras, como el modelado docente, las guías de procedimiento, los escenarios clínicos, entre otros.

Entre las anteriores técnicas, el modelado docente en simulación clínica consiste en la instrucción del paso a paso de un procedimiento clínico utilizando entrenadores por partes para explicar procedimientos asistenciales que luego el estudiante transferirá a la práctica profesional. La experiencia académica señala que el modelado docente debe ejecutarlo un docente experimentado, quien muestra en pequeños grupos la secuencialidad y la lógica de un procedimiento clínico-asistencial para afianzar el aprendizaje, personalizar la enseñanza y fomentar el aprendizaje autónomo.

En los estudiantes se ha fomentado este aprendizaje autónomo con la implementación de guías de procedimientos en simulación clínica, lo cual motiva al estudiante a ser protagonista de su aprendizaje. Las guias se han construido indicando el paso a paso acompañado de una ilustración fotográfica de diferentes procedimientos clínicos, como la punción venosa, el paso de una sonda de drenaje gástrico y el cateterismo vesical. Para Amaya, las guías de procedimiento permiten "organizar el pensamiento en torno a los procesos mentales y físicos implicados en el desarrollo de las habilidades y destrezas, lo cual favorece el aprendizaje significativo mediante la experiencia simulada" (2).

El aprendizaje guiado es uno de tipo experiencial que para Kolb, citado por Leal y cols. (3), está formado por cuatro etapas: experiencia concreta, observación reflexiva, conceptualización abstracta y experimentación activa. En la experiencia concreta, el estudiante precisa relacionarse con otros para aprender a tomar decisiones; en la observación reflexiva, el estudiante comprende significados de ideas y situaciones desde su propia perspectiva; en la conceptualización abstracta, aprende pensando, utiliza la lógica y planifica sistemáticamente, y en la experimentación activa, aprende actuando y utiliza la información nueva para influir en el ambiente. Las guías de procedimiento en simulación clínica propician experiencias tanto concretas como reflexivas, porque generan diálogo entre contenidos académicos y situaciones contextuales: a su vez, conectan el pensamiento lógico y sistemático con el conocimiento previo para lograr el aprendizaje significativo.

Las competencias enfermeras tanto técnicas como profesionales -incluidos los conocimientos, las habilidades y las actitudes-se adquieren en la formación profesional y se perfeccionan en espacios educativos en simulación clínica con la ayuda de las guías de procedimientos, las cuales se dinamizan en escenarios simulados que establecen relación con aprendizajes basados en problemas para ofrecer posteriormente solución 
contextual a situaciones que ocurrirán en la realidad. Según Díaz y cols. (4), el aprendizaje basado en problemas es una herramienta que, al ser incorporada en escenarios simples o complejos, permite evaluar las competencias profesionales e identificar el rol del estudiante, para conjugar la habilidad, el conocimiento, así como establecer actitudes mediante una mayor experiencia reflexiva.

Las guías de procedimiento se evalúan mediante listas de chequeo que permiten verificar las competencias tanto técnicas como profesionales y la identificación del rol, que junto con la experiencia, se incorporarán posteriormente a las prácticas en entornos reales. Estas "tienen el propósito de ofrecer al educando la oportunidad de realizar una práctica análoga a la que realizará en su interacción con la realidad en diferentes áreas o escenarios" (5).

La presente investigación muestra los resultados de un proceso de enseñanza y aprendizaje en estudiantes de tercer semestre de enfermería en una institución de educación superior de Bogotá, Colombia, a quienes se les aplicaron dos estrategias didácticas a través de la simulación clínica: 1) modelado docente y 2) guía de procedimiento para el aprendizaje autónomo. Esta última se construyó a partir de la revisión de la literatura, identificando varias competencias y los pasos secuenciales en un procedimiento clínico-asistencial (punción venosa). Las estrategias pretendían apoyar las competencias clínicas-asistenciales referidas a alistamiento de equipos, desarrollo secuencial de los pasos y registro enfermero. La guía fue evaluada con la lista de chequeo y el puntaje dicotómico, aplicado tras un entrenamiento autónomo bajo dinámicas como auto, hetero o coevaluación (6).

\section{Método}

El estudio se ubicó en el paradigma empírico, con enfoque cuantitativo, observacional y evaluativo. Utilizó como mecanismo de recolección de datos la evaluación en aula referida a la nota que obtuvo cada estudiante en su autoevaluación.

La población observada estuvo constituida por 175 estudiantes de tercer semestre de enfermería de una institución de educación superior, distribuidos en cinco periodos académicos distintos (1-2012 a 1-2014). ${ }^{5}$ Se trabajó con toda la población, por lo que no fue necesario realizar un cálculo para el tamaño muestral.

La unidad de análisis pretendió medir la efectividad en el aprendizaje para la punción venosa. Se compararon dos muestras poblacionales en quienes se aplicaron las estrategias didácticas (modelado docente = modelado y guía de procedimiento para el aprendizaje autónomo de la punción venosa

5. Razón por la cual encontraremos heterogeneidad en la población y muestras, semestre a semestre, evaluadas transversalmente. 
= guia). El instrumento utilizado para recolectar información fue una lista de chequeo diseñada por el equipo investigador. ${ }^{6}$

La investigación acogió los elementos contemplados en la Resolución 8430 de 1993, artículo 11, literal b. Por condiciones éticas, en la asignatura objeto de estudio, los estudiantes realizaban autónomamente y bajo orientación docente entrenamiento para el procedimiento de punción venosa. Se incluyeron todos los estudiantes que manifestaron de forma escrita su consentimiento de participar en el estudio, y se mantuvo en reserva su identidad. La lista de chequeo fue aplicada anónimamente; los estudiantes se beneficiaron en su aprendizaje mediante la retroalimentación obtenida de aquellos pasos del procedimiento donde presentaban individualmente dificultad.

Dentro del universo de evaluaciones - utilizando el modelado, la guía o una combinación de ambas - se buscó conocer qué tan exitosas han sido las diferentes metodologías de enseñanza. De acuerdo con esto, la hipótesis que surgió fue que los estudiantes que entrenaron con el modelado obtenían mejores resultados en sus calificaciones aplicadas la lista de chequeo de verificación, comparado con los que estudiaron mediante la guía o, por el contrario, estas son complementarias. Como hipótesis nula se determinó que no existe diferencia en los resultados entre ellas, frente a la suposición alternativa que se sustenta en que la estrategia de aprendizaje por medio del modelado es mejor que la guía.

Para la prueba de esta hipótesis se determinó un alfa de 0,05, y se escogió la prueba estadística F de Fisher (7), ya que las muestras de los dos grupos se llevan a cabo en tiempos transversales y son muestras independientes. La comparación contó con una distribución normal de la población y de la muestra.

Para la interpretación se tuvo en cuenta el error típico, el cual mostraba la variabilidad de los datos; entre tanto, el sustento se realizó verificando la desviación estándar, la varianza de la muestra y el rango estadístico, que tiende a ser más pequeño en aquella estrategia sobre la cual se surte mayor favorabilidad. Igualmente, a todos los grupos se les realizó la prueba de normalidad y de igualdad de varianza, con la que se identificaron el valor de p y el alfa.

\section{Resultados}

El comportamiento estadístico descriptivo en los cinco periodos académicos de observación (1-2012 a 1-2014) se registra en la tabla 1; la igualdad de varianza previa a la realización de la prueba de normalidad se ubica en la tabla 2. A continuación se amplía el comportamiento en cada periodo estudiado.

6. La lista de chequeo fue validada por 10 expertos temáticos. Está constituida por 42 ítems, cada uno valorado con puntaje dicotómico, que verifica los pasos consecutivos en la ejecución del procedimiento de punción venosa. 


\begin{tabular}{|c|c|c|c|c|c|c|c|c|c|c|c|c|c|c|c|c|}
\hline 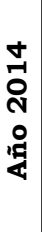 & 泀葋 & 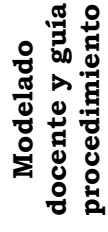 & 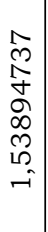 & $\begin{array}{l}0 \\
10 \\
0 \\
\infty \\
10 \\
م \\
0 \\
0\end{array}$ & $\begin{array}{l}10 \\
0 \\
- \\
-1\end{array} \mid$ & N & $\begin{array}{l}20 \\
0 \\
0 \\
0 \\
0 \\
0 \\
+ \\
0\end{array}$ & 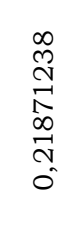 & $\begin{array}{l}\infty \\
\infty \\
\infty \\
1 \\
0 \\
0 \\
0 \\
0 \\
0\end{array}$ & 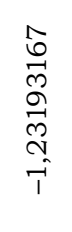 & $\begin{array}{l}0 \\
0 \\
-i\end{array}$ & $\left|\begin{array}{l}+ \\
0 \\
0 \\
0\end{array}\right|$ & N & $\begin{array}{l}\infty \\
+ \\
\infty \\
\infty \\
n\end{array}$ & $\stackrel{\infty}{m}$ & $\begin{array}{l}\infty \\
\infty \\
1 \\
1 \\
0 \\
10 \\
-1 \\
0\end{array}$ \\
\hline $\begin{array}{c}m \\
\stackrel{M}{-1} \\
\stackrel{N}{N}\end{array}$ & 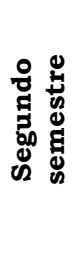 & 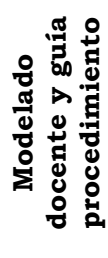 & $\begin{array}{l}\hat{N} \\
\text { م } \\
\text { N } \\
\text { જે } \\
\text { م } \\
\text { - }\end{array}$ & $\begin{array}{l}\infty \\
+ \\
\infty \\
\infty \\
0 \\
0 \\
1 \\
0 \\
0 \\
0\end{array}$ & $\stackrel{\infty}{-}$ & $\stackrel{\infty}{\rightarrow}$ & 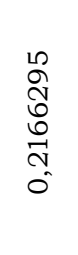 & 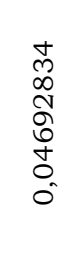 & $\begin{array}{l}\infty \\
\hat{0} \\
0 \\
0 \\
10 \\
0 \\
0 \\
0\end{array}$ & $\begin{array}{l}0 \\
\text { N } \\
0 \\
\circ \\
0 \\
0 \\
1 \\
\infty \\
0 \\
0 \\
1\end{array}$ & $\begin{array}{l}0 \\
0\end{array}$ & $\Rightarrow$ & N & $\begin{array}{l}0 \\
0 \\
0 \\
0\end{array}$ & ફે & $\begin{array}{l}0 \\
1 \\
N \\
N \\
N \\
0 \\
0 \\
0 \\
0\end{array}$ \\
\hline 量 & 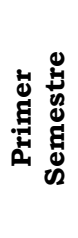 & 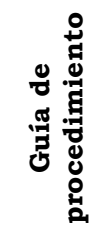 & 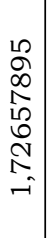 & $\begin{array}{l} \pm \\
0 \\
- \\
0 \\
0 \\
0\end{array}$ & $\begin{array}{l}\mathscr{L} \\
\stackrel{M}{2} \\
\stackrel{-}{-}\end{array}$ & $\stackrel{n}{\sim}$ & 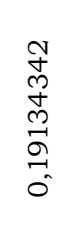 & 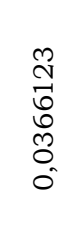 & $\begin{array}{l}m \\
0 \\
0 \\
0 \\
o \\
o \\
0 \\
0 \\
0 \\
i\end{array}$ & 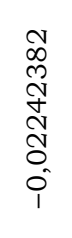 & $\begin{array}{l}0 \\
0\end{array}$ & 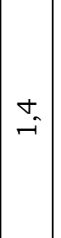 & N & $\begin{array}{l}\vec{b} \\
\hat{0}\end{array}$ & $\stackrel{\infty}{\infty}$ & 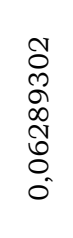 \\
\hline & 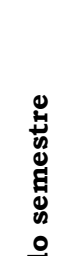 & 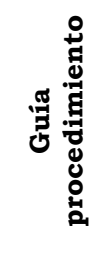 & $\begin{array}{l}0 \\
\stackrel{2}{r}\end{array}$ & $\begin{array}{l}0 \\
0 \\
0\end{array}$ & $\begin{array}{l}10 \\
\stackrel{2}{-} \\
-1\end{array}$ & $\begin{array}{l}\infty \\
\infty \\
- \\
-1\end{array}$ & $\begin{array}{l}F \\
0\end{array}$ & $\begin{array}{l}\tilde{0} \\
0 \\
0\end{array}$ & $\hat{n}$ & $\stackrel{\infty}{\stackrel{\infty}{\rightarrow}}$ & $\begin{array}{l}\mathcal{F} \\
\stackrel{5}{0} \\
0^{\prime}\end{array}$ & $\left|\begin{array}{l}0 \\
10 \\
-1\end{array}\right|$ & $\underset{-}{\stackrel{-}{-}}$ & $\begin{array}{l}\mathcal{T} \\
\hat{\sigma} \\
\hat{N}\end{array}$ & $\stackrel{n}{\sim}$ & $\begin{array}{l}0 \\
0 \\
0\end{array}$ \\
\hline $\begin{array}{l}\text { N } \\
\text { - }\end{array}$ & 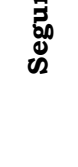 & 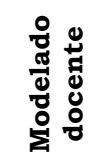 & $\begin{array}{l}\dot{J}_{-} \\
-i\end{array}$ & $\begin{array}{l}0 \\
0 \\
0\end{array}$ & $\begin{array}{l}0 \\
10 \\
-1\end{array} \mid$ & $\begin{array}{l}0 \\
\stackrel{1}{i} \\
-i\end{array}$ & $\begin{array}{l}\text { N } \\
\text { o } \\
0\end{array}$ & $\begin{array}{l}\text { L } \\
0 \\
0\end{array}$ & $\begin{array}{l}N \\
\tilde{n} \\
0 \\
1\end{array}$ & $\begin{array}{l}m \\
\stackrel{0}{0} \\
i\end{array}$ & $\begin{array}{l}10 \\
\stackrel{1}{0} \\
0\end{array}$ & $\begin{array}{l}8 \\
0 \\
-1\end{array}$ & 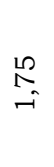 & $\begin{array}{l}\infty \\
i n \\
\vec{N} \\
-\hat{N}\end{array}$ & $\stackrel{n}{\sim}$ & $\stackrel{?}{\stackrel{2}{0}}$ \\
\hline 焉 & 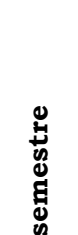 & 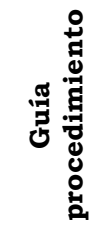 & $\begin{array}{l}0 \\
-i\end{array}$ & 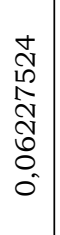 & $\begin{array}{c}10 \\
0 \\
- \\
-1\end{array} \mid$ & $\begin{array}{l}L \\
\infty \\
- \\
-\end{array}$ & \begin{tabular}{l}
0 \\
$L$ \\
0 \\
0 \\
$D$ \\
$\stackrel{1}{+}$ \\
\multirow{N}{N}{} \\
0 \\
0
\end{tabular} & \begin{tabular}{l}
$\hat{0}$ \\
0 \\
\multirow{J}{0}{} \\
0 \\
0 \\
0 \\
0
\end{tabular} & 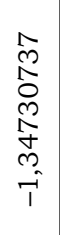 & \begin{tabular}{l}
$N$ \\
$N$ \\
$T$ \\
\multirow{T}{N}{} \\
0 \\
$N$ \\
0 \\
0 \\
0 \\
1
\end{tabular} & $\begin{array}{l}0 \\
0\end{array}$ & $\begin{array}{l}10 \\
N \\
- \\
-1\end{array} \mid$ & $\begin{array}{l}\infty \\
\infty \\
\rightarrow \\
-\end{array}$ & $\begin{array}{l}\infty \\
\text { ᄋ } \\
\stackrel{0}{0}\end{array}$ & $\stackrel{M}{\sim}$ & $\begin{array}{l}2 \\
0 \\
0 \\
\infty \\
0 \\
0 \\
10 \\
0 \\
0 \\
0\end{array}$ \\
\hline & 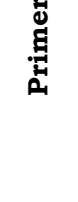 & 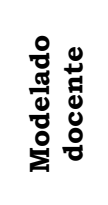 & $\begin{array}{l}\stackrel{0}{N} \\
\stackrel{N}{N} \\
\stackrel{-}{-}\end{array}$ & $\begin{array}{l}+ \\
0 \\
\infty \\
10 \\
\stackrel{2}{0} \\
0 \\
0 \\
0\end{array}$ & $\begin{array}{l}\stackrel{0}{\mathbb{N}} \\
\stackrel{\mathrm{N}}{\sim}\end{array}$ & $\begin{array}{l}\infty \\
\infty \\
- \\
-\end{array}$ & $\begin{array}{l}0 \\
\sim \\
D \\
\infty \\
\infty \\
ت \\
\vdots \\
0\end{array}$ & $\begin{array}{l}+ \\
0 \\
0\end{array}$ & 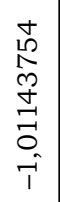 & 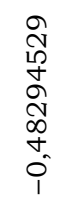 & \begin{tabular}{|l|}
10 \\
0 \\
0 \\
0
\end{tabular} & in & $\begin{array}{l}\infty \\
\infty \\
\rightarrow \\
-\end{array}$ & $\begin{array}{l}0 \\
\hat{N} \\
\stackrel{N}{n}\end{array}$ & $\stackrel{\bullet}{\sim}$ & 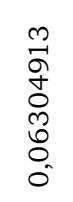 \\
\hline & 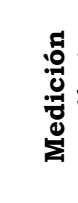 & & 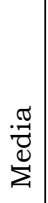 & 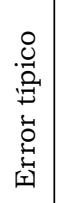 & 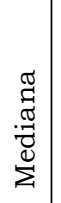 & $\begin{array}{l}\frac{\pi}{0} \\
0 \\
\Sigma\end{array}$ & 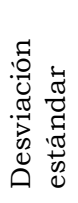 & 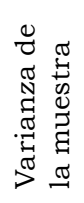 & $\begin{array}{l}\frac{n}{0} \\
0 \\
0 \\
0 \\
0 \\
0\end{array}$ & 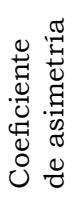 & 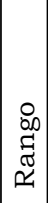 & 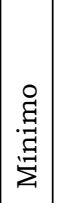 & 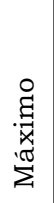 & 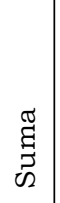 & 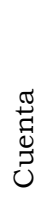 & 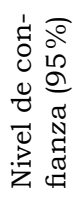 \\
\hline
\end{tabular}


Fanny Esperanza Acevedo Gamboa et al.

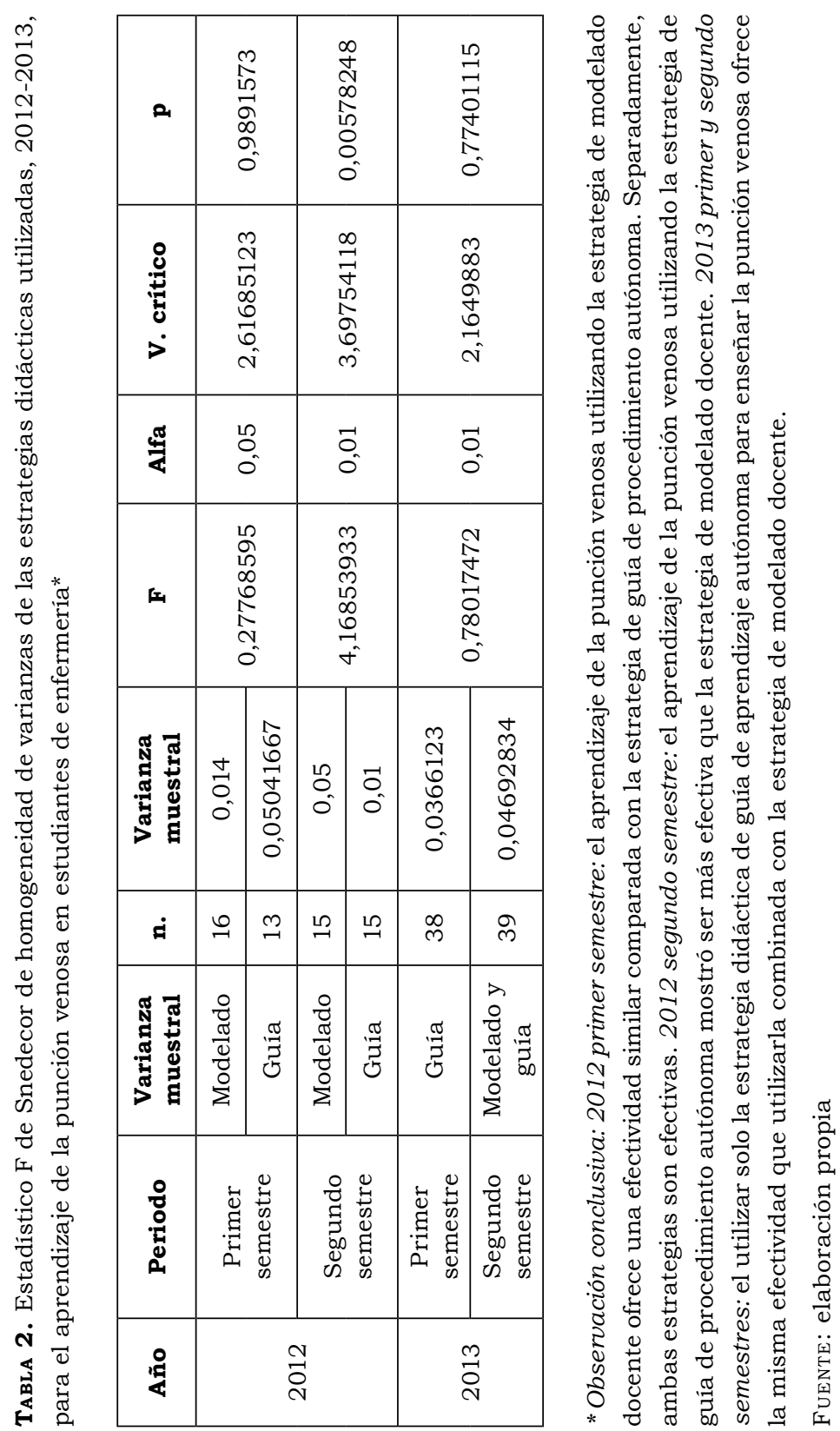




\section{Comportamiento de las estrategias didácticas para el aprendizaje enfermero de la punción venosa (periodos académicos 1-2012 al 1-2014)}

Primer semestre del 2012. Se compararon dos grupos: el primero con 16 estudiantes, a quienes se intervino con la estrategia de modelado docente, y el segundo grupo con 13 estudiantes, quienes participaron con la estrategia didáctica de guía de procedimiento para el aprendizaje autónomo de la punción venosa. Se identificó que el modelado docente tiene un mejor promedio $(1,72)$ en la nota de los estudiantes, comparado con la guía $(1,60)$. Sin embargo el test de Fisher (tabla 2) señala que no existe diferencia significativa entre los dos grupos respecto a la efectividad con la que fueron capacitados utilizando las dos estrategias.

Segundo semestre del 2012. Se repitió la experiencia con 30 estudiantes, distribuidos en dos grupos: el primero con 15 participantes intervenidos con la estrategia de modelado docente, y el segundo grupo con 15 participantes, intervenidos solo con la estrategia guía de procedimiento. Se identificó que la media de las evaluaciones (tabla 1) reflejaba que la estrategia de guía presentaba un mejor desempeño con un promedio de 1,76 , comparado con el modelado docente, que obtuvo 1,44. La prueba de igualdad de varianza (tabla 2) señaló que existe una diferencia significativa entre los grupos respecto a la efectividad con la que fueron capacitados, al señalar que la estrategia más representativa y que entregó mejores calificaciones como aprendizaje a los estudiantes es la guía.

Primer semestre del 2013. De acuerdo con la experiencia reportada en el segundo periodo académico del 2012, se optó por abandonar el modelado docente y se utilizó únicamente la guía de procedimiento. En esta oportunidad participaron 38 estudiantes. Se obtuvo una media de 1,72 con una desviación estándar de 0,19 y una varianza de 0,03 (tabla 1).

Segundo semestre del 2013. Se intervino a 39 estudiantes, combinando ambas estrategias didácticas (modelado docente y guía de procedimiento). Al comparar los resultados obtenidos en ambos periodos académicos del 2013, se identificó (tabla 1) que la estrategia de la guía tenía una media de 1,72 , en comparación con el 1,70 de la nota de los estudiantes del segundo periodo académico, en los que se combinó el trabajo de ambas estrategias (modelado docente y guía).

Primer semestre del 2014. Se valoraron 38 estudiantes, combinando las estrategias (modelado y guía). Se obtuvo una media de 1,53 con una desviación estándar de 0,467 y una varianza de 0,21 (tabla 1). 


\section{Comparación del comportamiento de las estrategias didácticas para el aprendizaje enfermero de la punción venosa, por periodos}

Primer semestre del 2012. El test de Fisher (tabla 2) señala que no existe diferencia significativa entre los dos grupos respecto a la efectividad con la que fueron capacitados utilizando ambas estrategias.

Segundo semestre del 2012. La prueba de igualdad de varianza (tabla 2) señaló que existe una diferencia significativa entre los grupos respecto a la efectividad con la que fueron capacitados, al evidenciarse que la estrategia más representativa y que entregó mejores calificaciones fue la guía.

Año 2013. La prueba de igualdad de varianza (tabla 2) arrojó que no existe diferencia entre las pruebas y el uso combinado de las estrategias utilizadas.

Primer semestre del 2014. Al contrastar los resultados del 2014 con los obtenidos en el primer periodo del 2013, cuando se utilizó únicamente la estrategia de guía, se encontró que esta última tiene una media de 1,72, la cual supera la obtenida en el 2014, al combinar ambas estrategias. La prueba de igualdad de varianza (tabla 3) concluyó que no existe una diferencia significativa en los resultados comparados para ambos grupos, lo que implica que utilizar solo la estrategia didáctica de la guía de procedimiento para el aprendizaje autónomo de la punción venosa ofrece la misma efectividad que combinarla con la estrategia de modelado docente.

TABla 3. Estadístico $\mathrm{F}$ de Snedecor de homogeneidad de varianzas de las estrategias didácticas utilizadas, 1-2013 y 1-2014, para el aprendizaje de la punción venosa en estudiantes de enfermería*

\begin{tabular}{|c|c|c|c|c|c|c|c|c|}
\hline Año & Periodo & $\begin{array}{c}\text { Estrategia } \\
\text { didáctica }\end{array}$ & $\mathbf{n}$ & $\begin{array}{c}\text { Varianza } \\
\text { muestral }\end{array}$ & F & Alfa & V. critico & $\mathbf{p}$ \\
\hline 2013 & $\begin{array}{c}\text { Primer } \\
\text { semestre }\end{array}$ & Guía & 38 & 0,014 & & & \\
\cline { 2 - 7 } 2014 & $\begin{array}{c}\text { Primer } \\
\text { semestre }\end{array}$ & $\begin{array}{c}\text { Modelado y } \\
\text { guía }\end{array}$ & 38 & 0,0366123 & 0,1673993 & 0,01 & 2,18052261 & 0,99999983 \\
\hline
\end{tabular}

*Observación conclusiva: no existe una diferencia significativa en los resultados comparados ambos grupos, o sea que el utilizar solo la estrategia didáctica de guía de aprendizaje autónoma para enseñar la punción venosa ofrece la misma efectividad que utilizarla combinada con la estrategia de modelado docente.

Fuente: elaboración propia

\section{Comparación del comportamiento estadístico por estrategias didácticas}

Con la información obtenida entre el 2012 y el 2014 se quiso determinar cuál estrategia didáctica era la más efectiva para el aprendizaje de la punción venosa en estudiantes de enfermería. En la tabla 4 aparecen dos comparaciones: en la primera se confrontan los resultados entre la estrategia de la guía frente al modelado docente. Allí se observó que el promedio de las evaluaciones obtuvo para la guía 1,70; en tanto que el modelado fue de 1,58. 
De acuerdo con la prueba de homogeneidad de varianzas, no existe diferencia significativa en los resultados, por lo que ambas estrategias son igualmente efectivas (tabla 5).

En la segunda comparación (tabla 4) se quiso determinar la efectividad en el aprendizaje, utilizando exclusivamente la guía de procedimiento contra el uso combinado de esta estrategia, incluido el modelado. Para normalizar los datos, y su posterior comparación, se eliminaron 15 de estos, y se pasaron de 77 a 62.7 La media de las evaluaciones refleja un mejor promedio de 1,72 para la guía, comparada la combinación de esta estrategia con la del modelado docente de 1,62; sin embargo, según las pruebas de igualdad de varianza, se concluyó que no existe diferencia significativa de los resultados finales, siendo estas igualmente efectivas (tabla 5).

TABla 4. Comportamiento estadístico comparado de la estrategia didáctica guía vs. modelado docente y estrategia combinada para el aprendizaje de la punción venosa en estudiantes de enfermería, 2012-2014

\begin{tabular}{|c|c|c|c|c|}
\hline \multirow{2}{*}{$\begin{array}{c}\text { Medición } \\
\text { estadistica }\end{array}$} & $\begin{array}{c}|c| \\
\text { Guía } \\
\text { procedimiento }\end{array}$ & $\begin{array}{c}\text { Modelado } \\
\text { docente }\end{array}$ & $\begin{array}{c}\text { Guia } \\
\text { procedimiento }\end{array}$ & $\begin{array}{c}\text { Modelado } \\
\text { docente y guía } \\
\text { procedimiento }\end{array}$ \\
\hline Media & 1,709494949 & 1,58655914 & 1,709494949 & 1,62519481 \\
\hline Error típico & 0,023311927 & 0,04057104 & 0,023311927 & 0,04222479 \\
\hline Mediana & 1,75 & 1,6 & 1,75 & 1,7 \\
\hline Moda & 1,5 & 1,5 & 1,5 & 1,8 \\
\hline $\begin{array}{c}\text { Desviación } \\
\text { estándar }\end{array}$ & 0,189386994 & 0,22588998 & 0,189386994 & 0,37052101 \\
\hline $\begin{array}{c}\text { Varianza de } \\
\text { la muestra }\end{array}$ & 0,035867433 & 0,05102628 & 0,035867433 & 0,13728582 \\
\hline Curtosis & $-0,570231502$ & 0,71979189 & $-0,570231502$ & 2,96423765 \\
\hline $\begin{array}{c}\text { Coeficiente } \\
\text { de asimetria }\end{array}$ & $-0,42057132$ & $-1,01777853$ & $-0,420057132$ & $-1,69544774$ \\
\hline Rango & 0,75 & 0,85 & 0,75 & 1,66 \\
\hline Mínimo & 1,25 & 1 & 1,25 & 0,34 \\
\hline Máximo & 2 & 1,85 & 2 & 2 \\
\hline Suma & 112,8266667 & 49,1833333 & 112,8266667 & 125,14 \\
\hline Cuenta & 66 & 31 & 66 & $77(62)^{*}$ \\
\hline $\begin{array}{c}\text { Nivel de } \\
\text { confianza } \\
\text { (95\%) }\end{array}$ & 0,046557134 & 0,08285711 & 0,046557134 & 0,08409795 \\
\hline
\end{tabular}

* La normalización requirió la eliminación de 15 datos para generar procesos de comparación, por lo que se pasó de 77 iniciales a 62.

FuEnTE: elaboración propia

7. Al tener una desviación superior a 3 desviaciones estándar, se procedió a eliminar los datos. 
Tabla 5. Estadístico F de Snedecor de homogeneidad de varianzas de las estrategias didácticas guía vs. modelado docente y estrategia combinadas, para el aprendizaje de la punción venosa en estudiantes de enfermeria, 2012-2014*

\begin{tabular}{|c|c|c|c|c|c|c|}
\hline $\begin{array}{c}\text { Estrategia } \\
\text { didáctica }\end{array}$ & $\mathbf{n}$ & $\begin{array}{l}\text { Varianza } \\
\text { muestral }\end{array}$ & $\mathbf{F}$ & Alfa & V. critico & $\mathbf{p}$ \\
\hline Guía & 66 & 0,03586743 & \multirow{2}{*}{1,42263551} & \multirow{2}{*}{0,01} & \multirow{2}{*}{2,00217544} & \multirow{2}{*}{0,11829958} \\
\hline Modelado & 31 & 0,05102628 & & & & \\
\hline Guía & 66 & 0,03586743 & \multirow[b]{2}{*}{0,26126103} & \multirow[b]{2}{*}{0,01} & \multirow[b]{2}{*}{1,81412245} & \multirow[b]{2}{*}{0,99999988} \\
\hline $\begin{array}{c}\text { Modelado y } \\
\text { Guía }\end{array}$ & 62 & 0,13728582 & & & & \\
\hline
\end{tabular}

* Observación conclusiva. Guía-modelado: no existe una diferencia significativa entre los que se capacitaron modelado docente en relación a los que solo se capacitaron utilizando la guía de procedimiento autónomo. Guía-modelado y guía: no existe una diferencia significativa entre los que se capacitaron combinando el modelado docente y el uso de la guía de procedimiento autónomo en relación con los que solo se capacitaron utilizando la guía de procedimiento autónomo.

Fuente: elaboración propia

\section{Discusión}

La experiencia educativa del grupo investigador indicaba que la estrategia de modelado docente para la enseñanza de la punción venosa surtía mejor efecto en el aprendizaje que utilizar una estrategia para el aprendizaje autónomo denominada guía. Tal hipótesis se quiso confirmar en la primera fase del estudio para el primer semestre del 2012; sin embargo, se comprobó estadísticamente que no existió diferencia entre estas, por lo que ambas estrategias permitieron generar habilidad y destreza en los estudiantes en relación con el cumplimento del mayor número de pasos de la lista de chequeo y no al éxito o fracaso del resultado del procedimiento.

Cuando se repitió la experiencia en el segundo semestre del 2012, los resultados mostraron que la guía estadísticamente fue más efectiva para el aprendizaje. Al establecerse la duda respecto a cuál estrategia didáctica mostraba mayor efectividad, en el primer semestre del 2013 se realizó la intervención con la guía de procedimiento y en el segundo semestre de ese año se combinó tal estrategia con el modelado. Los resultados para entonces indicaban que ambas estrategias eran efectivas usándose combinadas o de forma independiente.

Reunidos los datos de la experiencia de los cinco periodos académicos desde el primer semestre del 2012 hasta el primero del 2014, no existía diferencia significativa entre una y otra estrategia didáctica. Estas, de forma independiente o combinada, fueron efectivas para desarrollar habilidades y destrezas operativas para la punción venosa. Sin embargo, se identificó que la guía de autoaprendizaje podría llegar a ser más significativa para el aprendizaje. Con estas se promueve el desarrollo de habilidades cognitivas y operativas $(8,9)$; su uso dinamiza el proceso educativo, reduce tiempos y movimientos; incrementa el pensamiento crítico, y nunca reemplaza 
la labor docente ni la práctica clínica (10). A su vez, estas guías favorecen la seguridad en la atención de las personas en un sistema de salud complejo y establece respeto por la dignidad humana y la gestión del cuidado $(11,12)$.

El conocimiento construido por modelado docente en el aula de clase o en simulación clínica demanda una visión sociorrelacional para comprender la interacción con la realidad (13). La simulación clínica ofrece la oportunidad de llevar a cabo procedimientos cuantas veces sea posible, permite aprender del error y, ante todo, desarrollar aprendizajes significativos dentro de un modelo constructivista, porque se conecta los conocimientos previos con las realidades experienciales para establecer aprendizajes nuevos (14). La didáctica pretende solucionar problemas prácticos construyendo conocimiento vivencial o simulado que apoyara el aprendizaje al confrontarlo con la realidad $(15,16)$.

El diálogo establecido entre el estudiante y su aprendizaje se produce por repetición, ensayo-error, auto y coevaluación. Al respecto, Brydges (17) indica que las guias de autoaprendizaje benefician al estudiante, porque incrementan su autonomía y control de condiciones prácticas que satisfacen sus necesidades educativas. Las guías de aprendizaje autónomo son herramientas didácticas que apoyan la docencia, porque los estudiantes construyen significados, aprenden a ser sujetos comprometidos, asumen responsabilidades y aprenden a aprender $(10,11)$. Salvador y Gallego (18) han señalado que el instrumento didáctico de mayor importancia para desarrollar el trabajo autónomo es la guía de aprendizaje. La enseñanza de enfermería se beneficia de estas didácticas, porque incrementa la capacidad de confrontar con la realidad y desarrollar competencias que aseguren la función asistencial $(19,20)$.

\section{Conclusiones}

Las dos estrategias utilizadas (modelado y guía) favorecieron el aprendizaje del procedimiento de punción venosa; sin embargo, la estrategia guía de procedimiento ofreció la misma efectividad al ser utilizada de manera exclusiva o combinada con la estrategia modelado docente, esto a pesar que la estrategia de la guía, estadísticamente, mostró mayor desempeño.

El procedimiento de punción venosa es una técnica sencilla, cuya ejecución depende de múltiples variables tanto intrínsecas del paciente como extrínsecas a este; sin embargo, se quiso determinar la influencia de la estrategia didáctica en el aprendizaje en relación con el cumplimiento en el número de pasos y no en el éxito de la técnica, puesto que se considera que las estrategias utilizadas actúan como medio para el aprendizaje y no como resultado final del procedimiento. En esta investigación se estimuló el autoaprendizaje para realizar la punción venosa, el estudiante aprendió a su ritmo, se enfrentó con una realidad simulada, y las estrategias didácticas utilizadas (modelado y guía) demostraron ser efectivas y no excluyentes. Conviene continuar investigando en las prácticas educativas para enfermería utilizando guías para el autoaprendizaje de procedimientos clinicos asistenciales para determinar su efectividad en el desarrollo de competencias más especificas. 


\section{Financiamiento}

Ninguno

\section{Conflicto de intereses}

Los autores declaran no tener conflicto de intereses.

\section{Referencias}

1. Achury D. Estrategias pedagógicas en la formación de profesionales de enfermería. Investig Enferm. Imagen Desarr. 2008;10(2):3-6.

2. Amaya A. Importancia y utilidad de las "Guías de simulación clínica" en los procesos de aprendizaje en medicina y ciencias de la salud. Univ. Méd. 2011;52(3):309-14.

3. Leal C, Díaz J, Rojo A, Juguera M. Practicum y simulación clínica en el Grado en Enfermería, una experiencia de innovación docente. Universidad Católica de Murcia (UCAM) España. REDU. Revista de Docencia Universitaria [internet]. 2014 [citado 2015 jul 15];12(2):42151. Disponible en: http://red-u.net/redu/index.php/REDU/article/ view/606

4. Díaz JL, Leal C, García JA. Metodología de autoaprendizaje en entornos simulados (MAESC). Evidentia [internet]. 2014 [citado 2015 jun 22];11(45). Disponible en: http://www.index-f.com/evidentia/n45/ ev9620.php.

5. Gutiérrez I. La simulación clínica como herramienta de evaluación de competencias en la formación de enfermería. Reduca [Internet]. 2010;2(1):549-80. Disponible en: https://metodoinvestigacion.files. wordpress.com/2014/11/simulacic3b3n-clc3adnica-como-herramienta-de-evaluacic3b3n-de-competencias-inmaculada-de-la-horra.pdf

6. Rozas MR, Costa J, Francés L, Viñas H, Paulí A, Martínez C. Autoevaluación del aprendizaje en enfermería con ejercicios interactivos del programa Hot Potatoes. Educ Méd [Internet]. 2008 [citado 2015 jul 15];11(1):19-27. Disponible en: http://scielo.isciii.es/scielo. php?script $=$ sci_arttext\&pid $=$ S1575-18132008000100005\&lng=es.

7. Rustom J. Estadística descriptiva, probabilidad e inferencia una visión conceptual y aplicada [Internet]. 2012 [citado 2015 jul 15]. Disponible en: http://www.libros.uchile.cl/236.

8. Amaya A. Simulación clínica y aprendizaje emocional. Rev Colomb Psiquiatr [Internet]. 2012 [citado 2015 jul 15];41(Supl 2012):44-51. Disponible en: http://www.scielo.org.co/pdf/rcp/v41s1/v41s1a06.pdf.

9. Bux A. Nurses' perceptions of the usefulness of high fidelity simulation technology in a clinical education program. [Internet]. Ann Arbor: University of Phoenix; 2009 [citado 2015 nov 12]. Disponible en: http://proquest.umi.com/pqdweb?did=1748714651\&Fmt=7\&client $\mathrm{Id}=23922 \& \mathrm{RQT}=309 \& \mathrm{VName}=\mathrm{PQD}$.

10. Romero López MA, Crisol Moya E. Las guías de aprendizaje autónomo como herramienta didáctica de apoyo a la docencia. Escuela Abierta 
[internet]. 2012 [citado 2015 nov 12];15:9-31. Disponible en: file://D:/ Documentos\%20Perfil/Descargas/Dialnet-LasGuiasDeAprendizajeAu tonomoComoHerramientaDidact-4078711.pdf.

11. Huber G. Aprendizaje activo y metodologias educativas. Revista de Educación [Internet]. 2008 [citado 2015 feb 18];(número extraordinario):59-81. Disponible en: http://www.revistaeducacion.mec.es/ re2008/re2008_04.pdf.

12. Riancho J, Maestre J, Del Moral I. Simulación clínica de alto realismo: una experiencia en el pregrado. Educ Med [Internet]. 2012 [citado 2015 jul 15];15(2):109-15. Disponible en: http://scielo.isciii.es/pdf/ edu/v15n2/original4.pdf.

13. Montes de Oca N, Machado E. Estrategias docentes y métodos de enseñanza aprendizaje en la educación superior. Rev Hum Med [Internet]. 2011 [citado 2015 ene 22];11(3):475-88. Disponible en http://scielo.sld. $\mathrm{cu} /$ scielo.php?script=sci_arttext\&pid=S1727-81202011000300005.

14. Juguera L, Díaz JL, Pérez ML, Leal C, Rojo A, Echevarría P. La simulación clínica como herramienta pedagógica: percepción de los alumnos de Grado en Enfermería en la UCAM (Universidad Católica San Antonio de Murcia). Enfermería Global [Internet]. 2014 [citado 2015 jul 15];33:175-90. Disponible en http://scielo.isciii.es/pdf/eg/v13n33/ docencia3.pdf.

15. Morales C, Utili F. Rol de la simulación clínica en la seguridad del paciente [Internet]. 2012 [citado 2015 jul 15]. Disponible en: http://www.asepur.org/wp-content/uploads/2014/06/Rol-de-la-simulacio\%CC\%81ncli\%CC\%81nica-en-la-seguridad-del-paciente1.pdf.

16. De Jesús MI, Méndez R, Andrade R, Martínez DR. Didáctica: docencia y método. Una visión comparada. RTDCS Revistas de Teoría y Didáctica de las Ciencias Sociales. 2007;12(12):9-29.

17. Brydges RN. A critical reappraisal of self-learning in health professions education: Directed self-guided learning using simulation modalities [Internet]. Ann Arbor: University of Toronto; 2011 [citado 2015 nov 12]. Disponible en: http://proquest.umi.com/pqdweb?did=206903 0261\&Fmt=7\&clientId=23922\&RQT=309\&VName=PQD

18. Salvador F, Gallego JL. Enfoque didáctico para la individualización. La Didáctica General. [Internet]. 2003 [citado 2015 nov 12];247-72. Disponible en: http://lasonrisadejf.blogspot.com.co/p/capitulo-ix.html.

19. Galán G. Modelo conceptual de enfermeria, su relación de la ética y la bioética del cuidado con la persona y su dignidad. Enf Neuro México [internet]. 2012 [citado 2015 ene 22];11(2):91-7. Disponible en: http:// www.medigraphic.com/pdfs/enfneu/ene-2012/ene122g.pdf.

20. Clark DA. Nursing faculty perceptions on teaching critical thinking [Internet]. Ann Arbor: Capella University; 2010. Disponible en: http:// proquest.umi.com/pqdweb?did=1957434721\&Fmt=7\&clientId=23922\& $\mathrm{RQT}=309 \& \mathrm{VName}=\mathrm{PQD}$. 